\title{
Spatiotemporal distributions of rare bacterioplankton populations indicate adaptive strategies in the oligotrophic ocean
}

\author{
Kevin L. Vergin ${ }^{1}$, Brad Done ${ }^{1}$, Craig A. Carlson ${ }^{2}$, Stephen J. Giovannoni ${ }^{1, *}$ \\ ${ }^{1}$ Department of Microbiology, Oregon State University, Corvallis, Oregon 97331, USA \\ ${ }^{2}$ Department of Ecology, Evolution and Marine Biology, University of California, Santa Barbara, California 93106, USA
}

\begin{abstract}
Spatiotemporal distributions of rare microbial taxa were examined in 384 samples from the Bermuda Atlantic Time-series Study (BATS) site, in the northwestern Sargasso Sea. Sequences were partitioned into 6 mutually exclusive sets based on abundance (abundant, rare, and very rare) and frequency of detection (frequent and infrequent). Analyses of variance for taxa that were frequently present, across all levels of abundance, demonstrated environmental filtering, indicating that gradients in environmental factors, such as season and depth, drive community assembly for rare taxa, as they do for abundant taxa. All abundant nodal taxonomic units (NTUs) had spatiotemporal periods of rarity, providing a clear demonstration of the role of fluctuating reproductive success in population dynamics, and the role of rare populations as seed banks. An inverse relationship between the number of rare taxa and physical stratification indicates that transport by mixing drives increased community diversity throughout most of the year. Populations of selected copiotrophic taxa varied in episodic patterns that were not tightly entrained to season and depth, indicating that these populations are not governed by the same rules of community assembly that apply to most other taxa and may be adapted to exploit infrequent, unknown disturbances. Overall, the findings support the perspective that the success of most rare populations was driven by the same fundamental patterns of spatiotemporal variation that drove the success of dominant populations, but also indicate potentially important roles for transport by mixing and atypical life histories in determination of community composition.
\end{abstract}

KEY WORDS: Bacterioplankton $\cdot$ Rare $\cdot$ Copiotroph BATS · PhyloAssigner · Nodal taxonomic unit · NTU · Pyrosequence

Resale or republication not permitted without written consent of the publisher

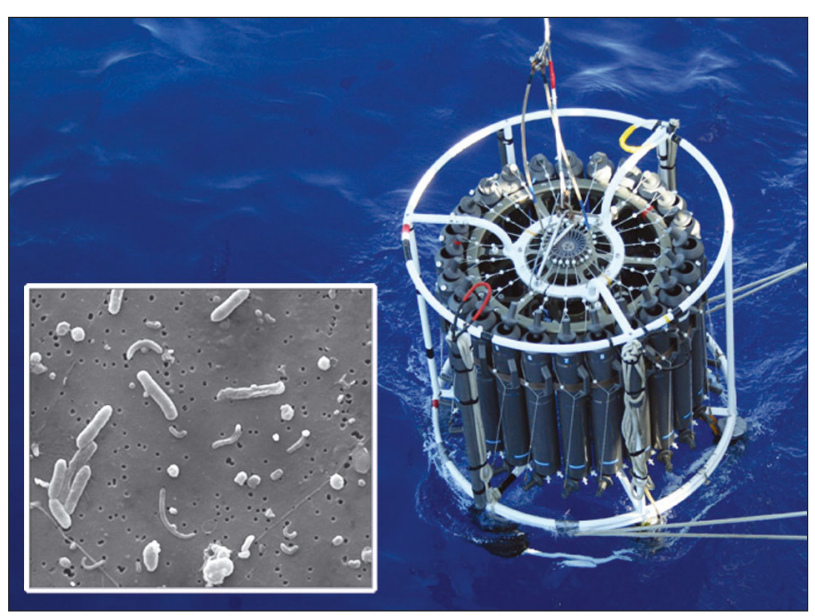

CTD array for sampling bacterioplankton (inset - scanning electron micrograph of a $40 \mathrm{~m}$ sample collected on a $0.2 \mu \mathrm{m}$ filter).

Photo: Craig Carlson; inset: Yanlin Zhao and the Oregon State University Electron Microscope Facility

\section{INTRODUCTION}

The Bermuda Atlantic Time-series Study (BATS) site, in the northwestern Sargasso Sea, is a seasonally oligotrophic marine environment (Steinberg et al. 2001). This region of the North Atlantic subtropical gyre is subject to cooling and high storm frequency in the winter months that results in deep convective mixing of the surface $300 \mathrm{~m}$. This mixing brings nutrients such as phosphorus and nitrogen into the euphotic zone, thus triggering blooms of photosynthetic microorganisms in the winter/early spring (January to April). As the storm frequency declines and water warms, it thermally stratifies in the late 
spring, resulting in distinct, stable bacterial communities in the mixed layer, the deep chlorophyll maximum, and the upper mesopelagic (Giovannoni \& Rappé 2000, Treusch et al. 2009). These communities persist from late spring through early autumn. During the summer and early fall, warm, stratified waters result in lower primary productivity (Menzel \& Ryther 1960, Steinberg et al. 2001, Nicholson et al. 2012). These low-productivity conditions are supported by regenerative production (Eppley \& Peterson 1979) dominated by picoplankton populations.

Theoretical issues surrounding the distributions of rare taxa in the oceans have been discussed (Sogin et al. 2006), but few microbial diversity surveys have had the sensitivity to address this issue. Pedrós-Alió (2012) speculated that rare taxa, represented by the long tails of rank abundance plots, represent seed banks that reproduce in response to favorable shifts in environmental conditions, and otherwise persist, aided by the advantage of diminished viral and zooplankton predation at low population densities. Brown et al. (2005) demonstrated that some taxa alternate between periods of rarity and abundance at the San Pedro Ocean Time Series study site. Campbell et al. (2011) showed that many taxa cycle between abundant and rare while others were always rare. Galand et al. (2009) showed that rare taxa can have a biogeographic distribution and are likely subject to selection, speciation, and extinction. As noted by Fuhrman (2009), some taxa rarely found in cloning studies are routinely cultivatable and other taxa may be rare, but nonetheless important to biogeochemical cycles. Fuhrman (2009) further suggested that high-throughput sequencing could be used to address hypotheses about microbial rarity.

Major features of marine bacterioplankton community structure have been characterized. Seasonality was demonstrated for community structure (Fuhrman et al. 2006, Caporaso et al. 2012, Gilbert et al. 2012) and specific taxa (Carlson et al. 2009, Treusch et al. 2009). Deep sequencing has suggested that some members of microbial communities are always present but vary in population size as environmental conditions vary (Caporaso et al. 2012) and that depth segregation, as well as seasonality at the surface and water mass segregation at depth, applies to both rare and abundant operational taxonomic units (OTUs; Galand et al. 2009). Community assembly processes for marine bacterial communities, however, are less well understood (Keddy 1992, Horner-Devine \& Bohannan 2006).

We considered 3 categories of rarity: (1) consistently present but rare in all samples; (2) abundant seasonally but persistent as rare members of the community at other times of the year; and (3) rare in most samples with spikes of abundance at irregular intervals. In all cases, perturbations are defined as a process whereby a disturbance of sufficient magnitude elicits a response in terms of altered densities or composition of species in a population or assemblage (Glasby \& Underwood 1996). Examples of disturbances at BATS include annual events, such as winter mixing, and sporadic events, such as eddies. Although winter mixing is seasonal, it is driven by deep mixing events that correspond to winter storms. These disturbances disrupt stratified populations and entrain nutrients such as nitrogen and phosphorus from deeper waters to the surface.

The definition of 'rare' is debated generally in ecology, where it is well recognized that plants and animals may be rare in one environment or sample and abundant in another (Pedrós-Alió 2012). For microbial samples, this debate also applies but is further complicated by the additional factor of time (Prosser et al. 2007). Microbes can respond to changing environmental conditions in a much shorter time period than plants or animals; thus, sampling frequency becomes a relevant factor in the determination of rarity. Taxa that are abundant in one season may be rare in another (Campbell et al. 2011). Additionally, the definition for bacterial species remains unresolved, making quantification especially difficult (Prosser et al. 2007). For the present study, we use the 0.1 to $1.0 \%$ range of relative abundance suggested by others (Pedrós-Alió 2006, Fuhrman 2009) as the definition of a 'rare' species and designate taxa $\geq 1.0 \%$ as 'abundant' and $<0.1 \%$ as 'very rare.' Additionally, we consider taxa present in $\geq 5.0 \%$ of samples to be 'frequent' and $<5 \%$ 'infrequent,' thus creating 6 mutually exclusive categories of abundance: frequent abundant (FA), infrequent abundant (IA), frequent rare (FR), infrequent rare (IR), frequent very rare (FVR), and infrequent very rare (IVR).

Some rare organisms are of particular interest. Enrichment experiments using seawater media and inocula from BATS resulted in rapid blooms of Alteromonas and other Gammaproteobacteria in response to the addition of glucose, ammonia, and phosphate (Nelson \& Carlson 2012). McCarren et al. (2010) saw a similar result with chemostat studies comparing amended seawater with unamended seawater from the Hawaii Ocean Time-series site in the Pacific Ocean. Suzuki et al. (1997) compared molecular analyses of an Oregon (USA) coast seawater sample with cultures isolated from the same water sample, many of which were Gammaproteobacteria, and 
confirmed the observations of many previous researchers that the cultures grown on rich media do not necessarily represent the abundant organisms in the natural population. These Gammaproteobacteria are clearly present in the water column but are at a difficult-to-determine low abundance and appear to respond rapidly to nutrient perturbations.

In this study, we used 16S rRNA pyrosequencing data from 384 samples collected monthly within the upper $300 \mathrm{~m}$ at the BATS site to characterize the bacterioplankton communities. Nodal taxonomic units (NTUs), in essence clades resolved to a fine scale, were used to define diversity (Vergin et al. 2013). Koeppel \& Wu (2013) have recently shown that ecological relationships emerge more clearly when phylogenetic diversity is resolved at finer scales. Our aim was to characterize taxon abundance in a long time series of samples and focus on rare taxa to identify recurring spatiotemporal patterns that might indicate niche specialization, especially with regard to seasonal cycling, depth stratification, and responses to disturbances.

\section{MATERIALS AND METHODS}

\section{Sample collection, nucleic acid isolation, PCR amplification, pyrosequencing procedures and data processing}

Details for sample collection and processing were described previously (Vergin et al. 2013). Briefly, in this study, 454 pyrosequencing data amplified from the V1 and V2 region of the 16S ribosomal RNA gene from 384 samples taken monthly at the BATS site (approximately $9 \mathrm{yr}$ of samples) were used. Samples were collected at the surface and $200 \mathrm{~m}$; we also collected an additional 35 depth profile samples (about $32 \%$ of the total number of months sampled) that included samples from 40, 80, 120, 160,250 , and $300 \mathrm{~m}$ (see Table S1 in the Supplement at www.int-res.com/articles/suppl/a071p001 _supp.pdf). For each sample, means of 6684 sequences with a mean of 257 bp length were generated. PhyloAssigner, a custom designed pipeline (Vergin et al. 2013) which incorporates the phylogenetic placement algorithm, pplacer (Matsen et al. 2010), grouped sequences into well defined phylogenetic groups, and a comprehensive backbone phylogenetic tree (http://aforge.awi.de/gf/project/ phyloassigner) was used to sort the sequence data into NTUs. NTUs are therefore similar to more familiar OTUs, except that NTUs are defined phylogenetically by reference sequences and clade structure rather than by cutoff thresholds. Although OTU binning using cutoff thresholds is computationally expedient, it has been demonstrated that phylogenetic placement using this method results in a loss of fine-scale phylogenetic information that can be important for resolving ecotypes (Koeppel \& $\mathrm{Wu}$ 2013, Vergin et al. 2013). For each sample, $>5000$ sequences were generated on average, allowing the relative abundance of populations to be measured to $0.1 \%$. The number of very rare NTUs (defined below) was not correlated to the total number of sequences in a given sample (Fig. S1 in the Supplement). The ecological software package PRIMER 6.0 (Clarke \& Gorley 2006) was used to process the data and provide statistical support for observations.

\section{Determination of seasonality}

Relative abundances for all samples were log transformed $(x+1)$ to improve homogeneity of variance, and a Bray-Curtis similarity matrix was generated. Physical measures were normalized and Euclidean distance was used to generate a sample similarity matrix. The decade of data was collapsed to a single composite year, all standardized to the timing of the annual deep convective mixing event as described by Carlson et al. (2009). The seasons were designated as follows: winter included the month prior, the month of, and the month after deep mixing. Spring and summer consisted of 3 mo groups after the winter months were designated, and autumn consisted of the remaining 1 to 4 mo (Carlson et al. 2009). A permutational multivariate analysis of variance (PERMANOVA) was conducted using season and depth designations as factors. PERMANOVAs were based on the Type III (partial) sum of squares and 999 permutations of residuals under a reduced model. Model matrices were also generated based on season or month designations for each sample by assigning equidistant values from 0 to 1 for the respective designations. Data matrices and model matrices were then compared using the Relate function and 999 random permutations. The Relate routine uses a Spearman rank correlation test to generate a coefficient, $\rho$, which measures the similarity of the rank order between 2 matrices of the same samples. A statistic is then derived empirically by comparing to values calculated after randomizing 1 of the matrices. Possible $\rho$ scores range from 1 (identical) to -1 (opposite), with random values clustered around 0 . 


\section{Diversity measures}

Diversity measures were calculated after first running Daisychopper (available from http://festinalente. me/bioinf/) to randomly generate equal numbers of sequences (1021) from each sample. Three samples generated fewer than 1021 sequences and were excluded from this analysis. Programming for estimates of Chao1 diversity (Chao 1984) was extracted from the SPADE website (Chao \& Shen 2010). Several different estimates of diversity (Ace, Chao-bc, Jackknife) were calculated, but the time-depth contour plots yielded similar results so only the Chao1 estimates are shown. PRIMER 6.0 was used to calculate Gini-Simpson and Shannon indices. Annual composite contour maps were generated using Ocean Data View software (http://odv.awi.de) in order to compare seasonal trends of the different parameters. Chao1 estimates, Gini-Simpson indices, and Shannon indices were compared using Student's $t$-tests. Gini-Simpson and Shannon indices were converted to true diversities (Jost 2006) prior to calculations. Chlorophyll a (chl a), chlorophyll $b$, and water temperatures were downloaded from the BATS website (http://bats.bios.edu/).

\section{Determination of rarity and frequency}

The entire dataset was partitioned into 6 sub-populations for comparison based on their level of abundance in the dataset and the frequency of their occurrence. NTUs were considered 'abundant' if they comprised $\geq 1 \%$ of the relative contribution within any sample, 'rare' if they were always $<1 \%$ but $\geq 0.1 \%$ in any sample, and 'very rare' if they were $<0.1 \%$ of the relative contribution in all samples. In addition, these categories were further divided by their frequency with a cutoff of $5 \%$ (where 'frequent' indicates presence [a non- 0 value] in $\geq 5 \%$ of samples and 'infrequent' indicates presence in $<5 \%$ of samples). Using only the NTUs from each category, Bray-Curtis similarities for samples were calculated and the resulting matrices were analyzed using the PERMANOVA function in PRIMER 6.0. Categories were mapped on the
PhyloAssigner reference tree using the Interactive Tree of Life software (Letunic \& Bork 2011).

Surface samples were sorted by season for comparisons of total and rare NTUs. For each sample, the number of NTUs with relative abundance $>0$ (total) and $<1.0 \%$ (rare) were enumerated. Values were compared using Student's $t$-test.

\section{RESULTS}

\section{BATS system depth and seasonal structuring}

Bray-Curtis similarities between all time-series and depth-series samples using all taxa were calculated and displayed in a non-metric multidimensional scaling plot, revealing patterns of variation in community composition with depth (Fig. 1A) and seasonal cycling (Fig. 1B). ANOVA and permutations of raw data (PERMANOVA) showed that the clustering of samples by depth and season were statistically significant (Table 1).

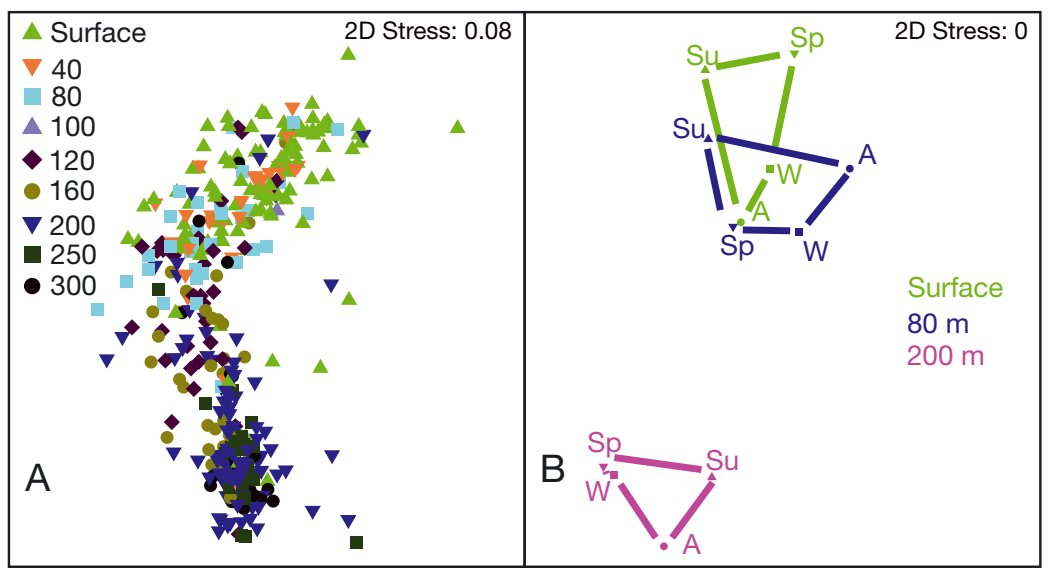

Fig. 1. (A) Non-metric multidimensional scaling (NMDS) plot of sample composition similarities calculated using all nodal taxonomic units (NTUs). Sample depths are distinguished by symbols as indicated. (B) Samples from the surface, $80 \mathrm{~m}$, and $200 \mathrm{~m}$ were averaged by season and plotted in an NMDS plot to highlight the seasonal cycling pattern. A: autumn, Sp: spring, Su: summer, W: winter

Table 1. Summary of PERMANOVA results for depth and seasonal partitioning in the full dataset. Perms: unique permutations

\begin{tabular}{|lcccccc|}
\hline Source & df & SS & MS & Pseudo- $F$ & p(perm) & Perms \\
\hline Depth & 8 & 237510 & 29689 & 30.644 & 0.001 & 999 \\
Season & 3 & 17570 & 5856.6 & 6.0449 & 0.001 & 998 \\
Depth $\times$ Season & 21 & 36639 & 1744.7 & 1.8008 & 0.001 & 996 \\
Residuals & 351 & 340070 & 968.85 & & & \\
Total & 383 & 651230 & & & & \\
\hline
\end{tabular}


Physical, chemical, and pigment data provided by the BATS core program (http://bats.bios.edu) were analyzed to uncover correlations between environmental factors and the observed spatiotemporal variation in microbial community composition. PERMANOVA of a sample similarity matrix made from these data revealed significant seasonality and depth partitioning (Table S2 in the Supplement), and the same matrix was significantly correlated to a matrix based on NTU abundance by the Relate routine $(\rho=0.231)$. However, a comparison of Spearman correlation values between the physical measurements and NTU abundance yielded no correlations that convincingly supported isolation of any particular environmental factor as a main driver of community structure. Overall, what we observed supported previous conclusions that seasonality and depth partitioning of microbial communities in the ocean surface layer are driven by a complex network of factors (Vergin et al. 2013), since no single factor can convincingly explain these patterns.

Similar analyses were applied to 6 subsets of the data, partitioned by frequency of occurrence and relative abundance (FA, IA, FR, IR, FVR, and IVR), to determine whether variation in the subsets of taxa followed similar patterns to the community as a whole. Presence in $\leq 5 \%$ of the samples was chosen subjectively as the frequency cutoff defining the boundary between frequent and infrequent. About $40 \%$ of all NTUs (514) were infrequent by this criterion (Table 2). Increasing cutoff thresholds at 5\% intervals increased the total number of infrequent NTUs by small increments $(10,15$, and $20 \%$ corresponded to 669,757 , and 829 NTUs, respectively); thus, the majority of infrequent NTUs were captured with the $5 \%$ cutoff. NTUs designated as FA, FR, IR, FVR, and IVR were distributed throughout the bacterial tree, with every major branch containing representatives of these subsets (Fig. S2). The IA subset consisted of 6 NTUs.

PERMANOVA of 3 subsets of taxa (FA, FR, and FVR) indicated significant spatiotemporal structuring (Tables $2 \&$ S3). The other 3 subsets (IA, IR, and IVR) were not significantly structured, with the exception of weak seasonal structuring of the IVR subset. However, some individual taxa within the IR and IVR groups were significantly related to model matrices based on either depth or season (Table 2). The number of data points in the 'frequent' subsets is generally much greater than the number of data points in the 'infrequent' subsets. To control for this potential bias, FA taxa were segregated and reduced to subsets of $5 \%$ frequency or less by converting the abundances to 0 in all but the 2.5 to $5 \%$ of months under consideration to simulate IA datasets. PERMANOVA of these simulated datasets still indicated significant spatiotemporal structuring (Table S4), thereby showing that structuring is not biased by a low percentage of samples with non-0 relative abundances. Populations of FR and FVR bacterioplankton displayed patterns of seasonality and vertical partitioning similar to FA populations, indicating that their reproductive success was also driven by adaptation to variables that change with season and depth.
Table 2. Depth and seasonal structuring within categories of taxa defined by combinations of occurrence frequency and relative abundance; see Table S3 in the Supplement at www.int-res.com/articles/suppl/a071p001_supp.pdf for detailed results of the PERMANOVA. NTU: nodal taxonomic unit; FA: Frequent (present in $\geq 5.0 \%$ of samples) Abundant ( $\geq 1.0 \%$ in any sample); FR: Frequent Rare (maximal abundance $\geq 0.1 \%$ but $<1.0 \%$ ); FVR: Frequent Very Rare (always $<0.1 \%$ ); IA: Infrequent (present in $<5.0 \%$ of samples) Abundant; IR: Infrequent Rare; IVR: Infrequent Very Rare; ND: not determined

\begin{tabular}{|c|c|c|c|c|c|}
\hline \multirow[t]{2}{*}{ Category } & \multirow[t]{2}{*}{$\begin{array}{l}\text { No. of } \\
\text { NTUs }\end{array}$} & \multicolumn{2}{|c|}{$\begin{array}{c}\text { PERMANOVA significant } \\
\text { correlation }\end{array}$} & \multicolumn{2}{|c|}{$\begin{array}{l}\text { No. of NTUs with significant } \\
\text { correlation to model }^{\mathrm{a}}\end{array}$} \\
\hline & & Season & Depth & Month & Depth \\
\hline FA & 240 & + & + & ND & ND \\
\hline FR & 480 & + & + & ND & ND \\
\hline FVR & 88 & + & + & ND & ND \\
\hline IA & 6 & - & - & 1 & 0 \\
\hline IR & 65 & - & - & 2 & 1 \\
\hline IVR & 443 & + & - & 1 & 8 \\
\hline
\end{tabular}

\section{Community diversity analyses}

Chao1, Gini-Simpson, and Shannon diversity measures (Jost 2010) were condensed into a composite year, aligned to the month of deepest mixing, and plotted by depth and month (Fig. 2). All 3 measures were strongly affected by the onset of deep mixing, a natural, annual disturbance, and remained perturbed until the water column began to stratify in late spring. Chao1 richness estimates (Fig. 2A) gradually increased in the surface layer as waters cooled (Fig. 2F) and winter mixing progressed to its maxi- 

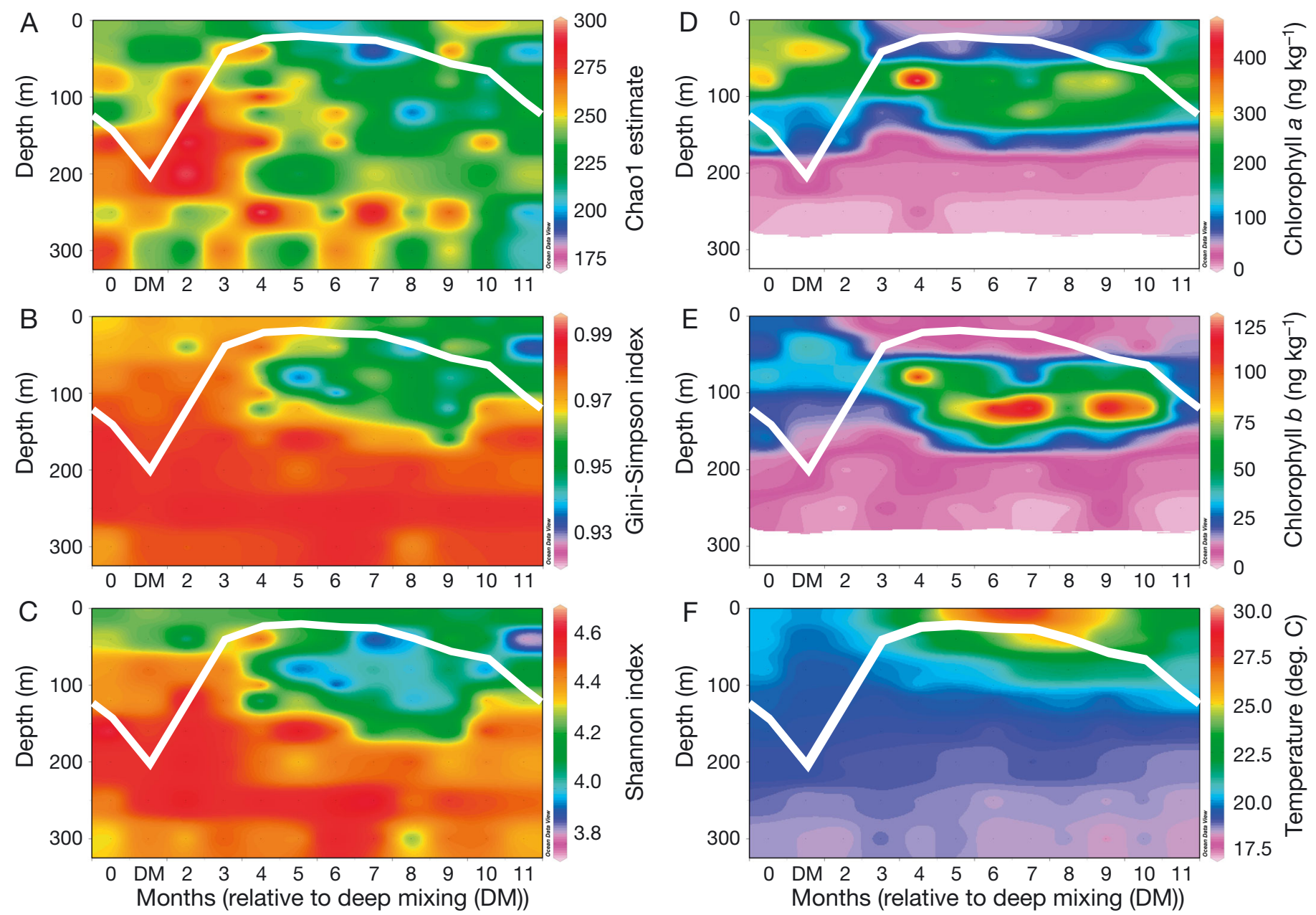

Fig. 2. Contour plots for (A) Chao1 estimates, (B) Gini-Simpson index values, (C) Shannon index, (D) chlorophyll (chl) $a$, (E) chl $b$, and (F) temperature for each sample plotted as an average over a 1 yr time span. The plots are indexed to the month of deepest mixing (DM) for each year, designated as month 1 ( $x$-axis). The white line represents the average mixed layer depth. Chl $a$ is associated with eukaryotic phytoplankton and chl b is associated with Prochlorococcus

mum depth, probably reflecting the mixing of surface and deep communities. Chao1 estimates returned to lower richness values in the summer as the water stratified. In late winter, as the deep mixing layer shoaled, there was a region of maximum richness extending from $80 \mathrm{~m}$ to over $200 \mathrm{~m}$, which is likely related to annual spring blooms of eukaryotic phototrophs (Fig. 2D). The contour plot of Gini-Simpson diversity indices (Fig. 2B) increased throughout the water column during deep mixing and returned to lower diversity values in the upper $120 \mathrm{~m}$ during the summer, as waters stratified and abundant microorganisms such as SAR11 reached their maximum (Morris et al. 2002). The contour plot of Shannon diversity indices (Fig. 2C) similarly indicated a shift from higher diversity during the deep mixing period to lower diversity during summer stratification. Student's $t$-tests confirmed that the observed variation in richness and diversity with depth was significant (comparison of surface and $200 \mathrm{~m}$, Chao1 $\mathrm{p}<0.0014$, Gini-Simpson $\mathrm{p}<<0.0001$, and Shannon $\mathrm{p}<<0.0001$ ), as suggested by an earlier study (Treusch et al. 2009). There were no significant differences in diversity measures from season to season.

Summer samples had the lowest number of total NTUs and rare NTUs and, in combination, a low proportion of rare NTUs (Table 3), which would be predicted since that region had lower richness and lower overall diversity. Total NTUs and rare NTUs increased significantly during the transition from summer to autumn, a period during which the mixed layer deepens, well before winter storms force deep mixing. Transport of cells by mixing is a plausible explanation for the inverse relationship between physical stratification and diversity that we observed, but an alternative explanation is that mixing sup- 
ports an increase in diversity, not because of transport, but because it creates more complex environments that support a wider range of adaptive strategies.

\section{Seasonal abundance and rarity}

Every FA and IA NTU in the dataset also had periods of rarity (data not shown). The percentage of samples where FA, FR, and FVR NTU abundance

Table 3. Average (SD) seasonal composition of total and rare nodal taxonomic units (NTUs) in surface samples. Bold indicates a significant Student's $t$-test difference compared to the previous season. ${ }^{*} \mathrm{p}<0.05,{ }^{* *} \mathrm{p}<0.01$

\begin{tabular}{|lcccc|}
\hline & Spring & Summer & Autumn & Winter \\
\hline Total NTUs & 162.2 & 152.7 & $\mathbf{1 6 7 . 2}$ & 170.5 \\
& $(20.7)$ & $(15.6)$ & $\mathbf{( 2 0 . 2 )}^{* *}$ & $(20.3)$ \\
Rare NTUs & 62.0 & 56.1 & $\mathbf{6 4 . 8}$ & 60.7 \\
& $(11.1)$ & $(8.7)$ & $\mathbf{( 1 1 . 6 )}^{*}$ & $(12.2)$ \\
Proportion of & 38.2 & 36.6 & 38.6 & $\mathbf{3 5 . 3}$ \\
rare NTUs & $(4.2)$ & $(3.0)$ & $(3.6)$ & $\mathbf{( 4 . 2 )}^{* *}$ \\
\hline
\end{tabular}
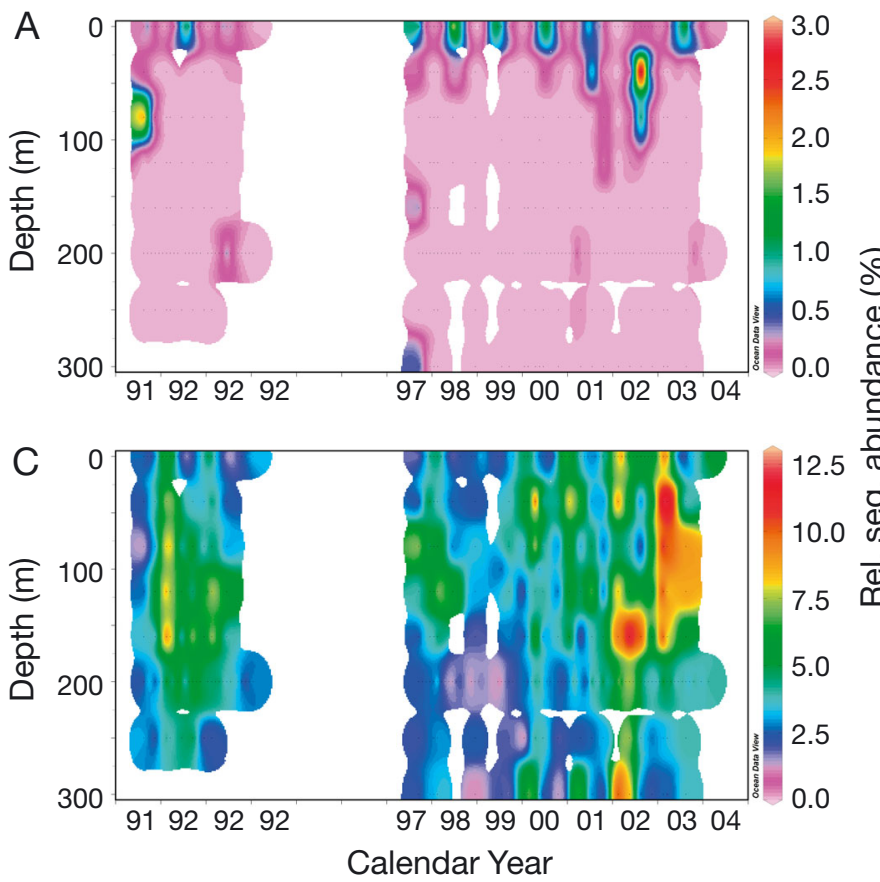

was within 1 SD of their mean was 80,66 , and $29 \%$, respectively, indicating that fluctuations in the reproductive success of individual NTUs is universal for this system. Most commonly, this pattern was observed for NTUs with systematic temporal or vertical trends (Fig. 3a,b). In the extreme case of consistently high abundance at many depths and times, for example NTU 1257, a deep branching representative of SAR11 subclade Ib (Fig. 3c), there were still instances of low abundance in the dataset. By contrast, the opposite case of very low abundance throughout the dataset except for 1 or a few samples when the NTU peaked was also observed as demonstrated by NTU 1731, a deep branching representative in the Bacteroidetes (Fig. 3d). Fluctuations in reproductive success for individual NTUs result in community turnover as successful taxa replace declining taxa.

\section{Episodic patterns in Gammaproteobacteria abundance}

Seventeen NTUs were detected that belong to the families Alteromonadaceae and Vibrionaceae.

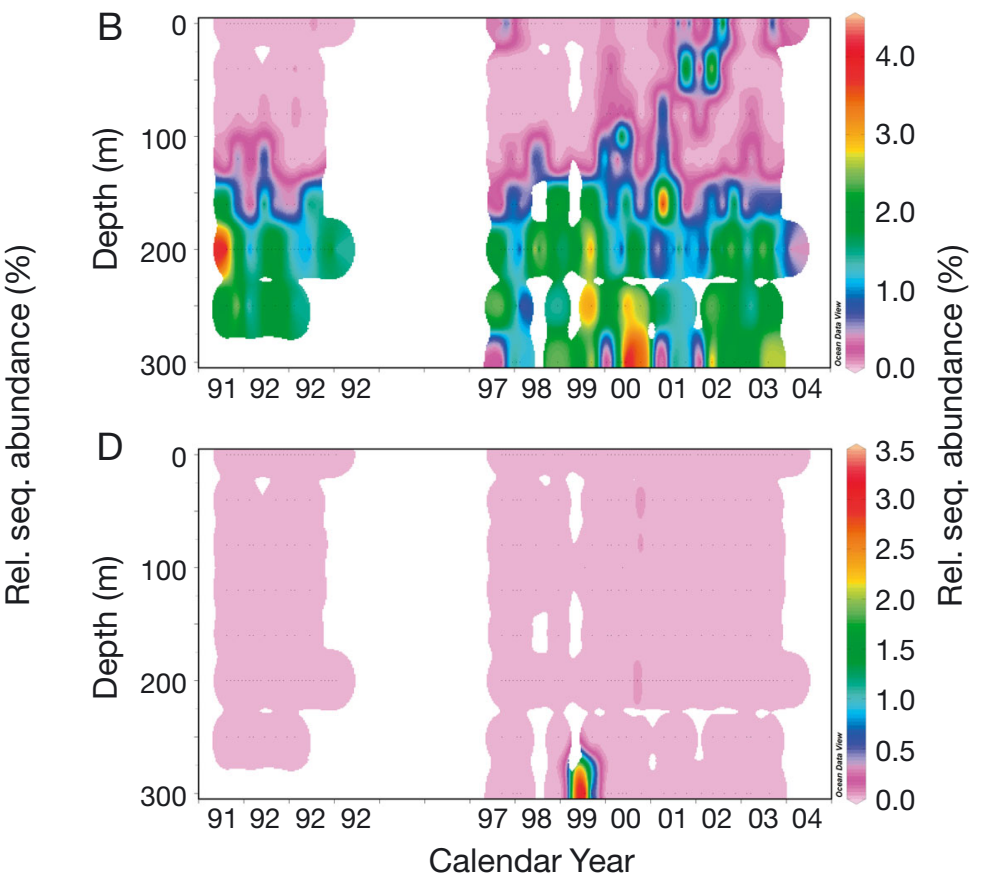

Fig. 3. Contour plots of the entire time series for (A) nodal taxonomic unit (NTU) 1117, a Frequent Abundant (FA; see definitions in Table 2) clade of SAR116 that is present annually in the upper euphotic zone, (B) NTU 2588, an FA clade of SAR202 group 1 that is primarily observed in the mesopelagic zone, (C) NTU 1257, an FA deep clade of SAR11 Ib that is abundantly present throughout the water column and during most times of the year but has some periods of rarity, and (D) NTU 1731, an Infrequent Abundant (IA) clade of Bacteroidetes that is rarely present in this dataset but is capable of periodic peaks in abundance when conditions are presumably favorable. These plots represent common patterns of abundance and rarity which are typical for abundant NTUs. Color bar intensities were adjusted to show maximum ranges of relative sequence (rel. seq.) abundance proportion 
These are well-known Gammaproteobacteria taxa that grow easily in culture but are rarely detectable in situ using molecular biology techniques (Carlson et al. 2004, McCarren et al. 2010, Nelson \& Carlson 2012). In 11 of the 17 cases, these NTUs were classified as IA, FR, IR, FVR, or IVR, indicating that they were consistently low abundance and/or low frequency. The remaining 6 FA NTUs were highly episodic, as seen in contour plots of the entire time series (Fig. 4). One time period in particular, around January 1998, was apparently favorable throughout the water column for several Vibrionaceae NTUs (Fig. 4A-D). The enhanced relative contribution of Vibrionaceae also corresponded to an unusual increase in concentration of chlorophyllide a (Fig. S3), an intermediate of the chlorophyll and bacteriochlorophyll biosynthetic pathways (Müller et al. 2011). It is not clear whether there is a direct relationship between pulses of high abundance in Vibrionaceae and the sharp increases in chlorophyllide a. At 3 other times (October 1991, October 1993, and December 2003), NTUs associated with Alteromonadaceae were elevated (Fig. 4E,F) at $200 \mathrm{~m}$. It is possible that our monthly sampling frequency was too coarse to adequately capture all events for these Gammaproteobacteria and other copiotrophic organisms, resulting in an apparently random distribution of population maxima throughout the time-series data. It is unlikely that these signals are due to sample handling because these Gammaproteobacteria were detectable at low levels in many samples, tended to
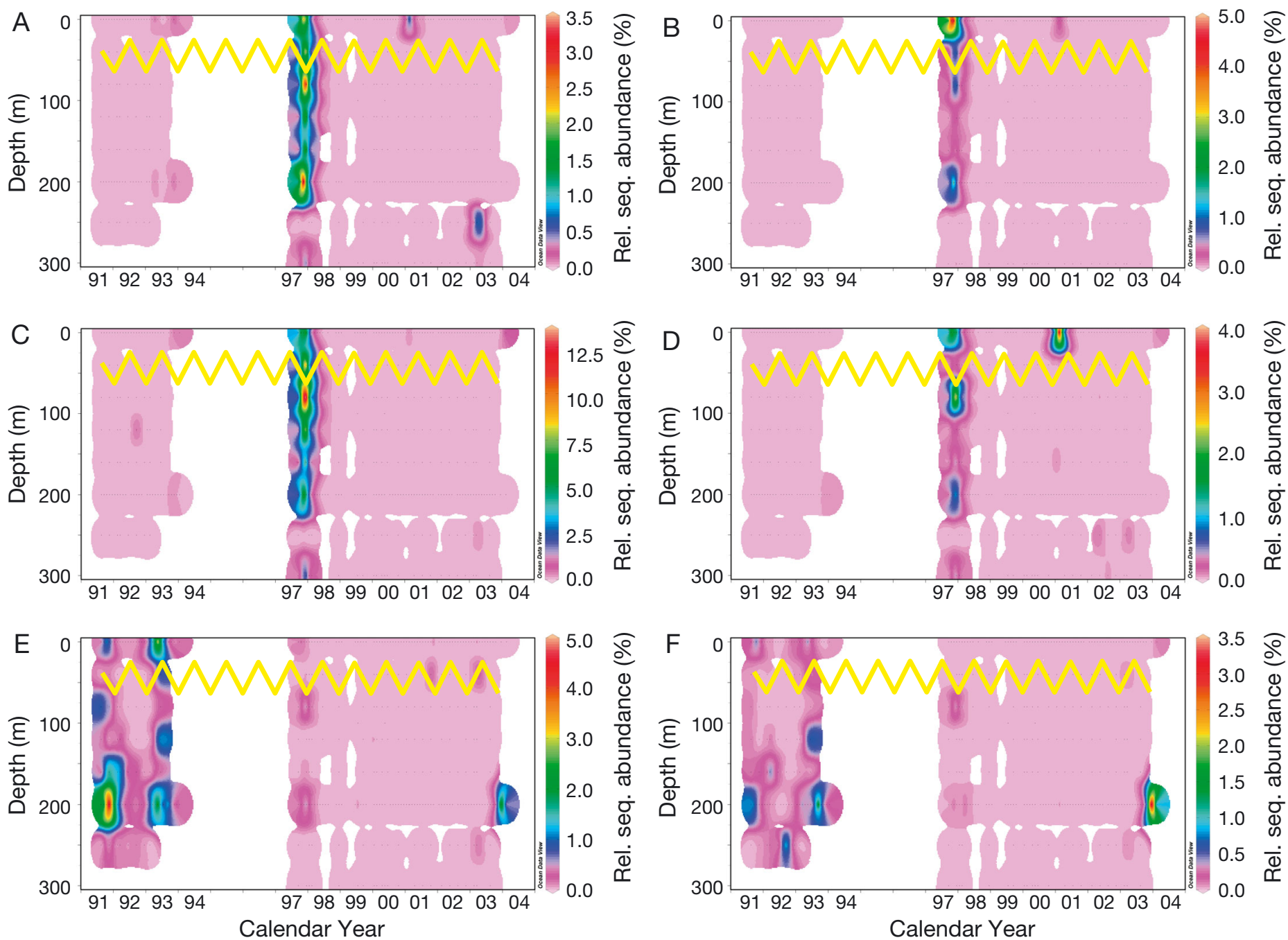

Fig. 4. Contour plots of the relative contribution to the total community of 4 Frequent Abundant (FA; see definitions in Table 2) Vibrionaceae-(A) nodal taxonomic unit (NTU) 52, (B) NTU 53, (C) NTU 61, and (D) NTU 63 - and 2 FA Alteromonadaceae-(E) NTU 77 and (F) NTU 83. Intensities were adjusted to show maximum ranges of relative sequence (rel. seq.) abundance proportion. The yellow line represents the average monthly sunlight ranging from $611 \mathrm{~min} \mathrm{~d}^{-1}$ in December to $848 \mathrm{~min}^{-1}$ in June 
be high in samples collected before and after the dates of their peak abundances, and were not present in PCR negative control samples.

Another method with which to measure episodic appearance is to calculate the difference between the maximum and mean abundance and compare those values to the $\mathrm{SD}$ of the abundance. These paired values are shown in a scatterplot for 246 FA NTUs (Fig. S4). The majority of NTUs clustered in a narrow range. However, the Vibrionaceae and Alteromonadaceae NTUs were at higher values along the axis representing the difference between the maximum and the mean but at the lower range of SD values. For comparison, NTU 614, a member of the Burkholderia, with a similar maximum-mean difference and SD to the episodic Vibrionaceae and Alteromonadaceae NTUs, is shown (Fig. S4), indicating that other taxa in this area of the plot were similarly episodic. Most of the Vibrionaceae and Alteromonadaceae NTUs were classified as rare, but analysis of the FA NTUs revealed the pattern of these groups. Due to their persistence in this oligotrophic system, these groups of copiotrophs appear to be capable of responding opportunistically to unknown stimuli.

\section{DISCUSSION}

In a dynamic system, such as BATS, annual cycles in chemical and physical conditions ensure that a combination of bottom-up and top-down mechanisms will select for different subsets of microbial diversity over the course of the year, causing predictable patterns of community turnover (Fuhrman et al. 2006, Carlson et al. 2009, Treusch et al. 2009, Caporaso et al. 2012, Gilbert et al. 2012). Environmental filtering (abiotic and biotic driven community assembly processes) give rise to annually repeated peaks of reproductive success and subsequent retreat to lower abundances for abundant taxa, which drives seasonal community turnover. In theory, at any given time, environmental filtering will select for a subset of NTUs, while other NTUs may be less active and serve as a seed bank until conditions change. Since conditions are not at a steady state, a majority of NTUs that are abundant in one spatiotemporal locus become rare in other seasons or depths, as has been reported in other systems (Brown et al. 2005, Campbell et al. 2011). Thus, at a single spatiotemporal locus, the community is, on average, composed of abundant and rare members, and the designation 'rare' is often dependent on the spatiotemporal locus of sampling.
Like abundant taxa, most rare NTUs are not randomly distributed, but vary in patterns that suggest their reproductive success is due to environmental filtering. Similar variation has been observed in dominant bacterioplankton populations (Morris et al. 2005, Fuhrman 2009, Campbell et al. 2011, Gilbert et al. 2012, Vergin et al. 2013). PERMANOVA showed that, as a group, FR and FVR populations varied seasonally and were partitioned by depth. It is not clear what physical, chemical, or biological environmental factors control the reproductive success of either rare or dominant taxa. One possibility is that the reproductive success of some rare taxa is tied to the reproductive success of other, perhaps more abundant taxa. In one hypothetical scenario, species-specific metabolites released by leaky membranes, viral lysis, or inefficient feeding by predators could be a source of nutrients for other cell populations. In an alternative scenario known as the 'Black Queen Hypothesis,' Morris et al. (2012) postulated that rare taxa could provide metabolic products that are critical to the reproductive success of more abundant taxa, providing a niche in the community that has its basis in co-evolutionary interactions. One potential advantage of maintaining a lower abundance is a lower vulnerability to viruses and grazer predation (PedrósAlió 2012). The relative importance of these issues remains unresolved, and the main point here is that most rare taxa do not vary independently in the data set we report, but are entrained to the environment, including the surrounding community, by unknown factors that control their success.

In contrast, IR and IVR NTUs displayed very little significant spatiotemporal patterning. The hypothesis that this finding might be an artifact of undersampling was not supported when tested by subsampling more frequent taxa to simulate similar, low sampling frequencies (Table S4). These observations suggest that the distributions of IR and IVR organisms are not controlled by environmental filtering. Several hypotheses could explain the lack of spatiotemporal patterning (Pedrós-Alió 2012 and references therein). Some of these taxa may be adapted to narrowly defined environmental conditions, particularly susceptible to viruses or predation, deposited from a different environment, encapsulated in spores, or in transit between favorable environments. It is possible that this oligotrophic ocean system is similar to lownutrient lakes (Lennon \& Jones 2011) where dormancy was a strategy utilized by about $40 \%$ of taxa. However, activity rates were not determined in this study, so partitioning of the rare taxa in the BATS community into active and dormant classes (Hugoni 
et al. 2013) is not possible here. We cannot rule out the possibility that responses of some taxa may be short lived ( $<1 \mathrm{mo})$ and therefore below the resolution of our sample collection frequency. Determination of the exact roles of rare organisms in these communities will require more investigation and may support multiple hypotheses as a range of survival strategies will likely emerge.

Predicted changes in ocean conditions may adversely affect rare taxa and have profound effects on community stability. The most important driver of community turnover at BATS is annual water column mixing, which dramatically changes nutrient gradients and relaxes predation pressures, causing phytoplankton blooms in a complex successional pattern, and microbial community turnover (Morris et al. 2005, Behrenfeld 2010, Treusch et al. 2012). Distinct trends in measurements of richness and diversity showed that, over the course of a year, these fundamental properties of the water column community change. As the depth of the mixed layer increased over the course of the autumn and winter, richness and diversity increased. In the spring, following deepest mixing, the community transitioned rapidly to become less rich and less diverse, with greater abundance of fewer NTUs. In the summer, richness and diversity in the euphotic zone reached their lowest levels as the surface layer stratified, nutrients were depleted (Steinberg et al. 2001), and the community shifted from one dominated by eukaryotic phytoplankton (Fig. 2D) to one dominated by Prochlorococcus (Fig. 2E) (DuRand et al. 2001, Treusch et al. 2012). The decrease in diversity in the summer months is driven by the reproductive success of a few populations, such as SAR11 subclade Ia (Carlson et al. 2009, Vergin et al. 2013), SAR116 (Treusch et al. 2009), and Prochlorococcus (Fig. 2E). It is theoretically possible that taxa rare in other seasons are not counted as rare in the summer because they become abundant in that season, but we saw no evidence for this case (data not shown). A positive correlation between richness and community stability has been demonstrated in macrobial (Chapin et al. 2000) and microbial communities (Naeem \& Li 1997, Morin $\&$ McGrady-Steed 2004). A similar correlation has also been shown between diversity and microbial community stability (Wittebolle et al. 2009). Climate change is causing warming of the ocean surface, increasing water column stratification, and decreasing chlorophyll over wide ocean expanses (Behrenfeld et al. 2006). This process, here referred to as ocean desertification, is predicted to widely shift ocean ecosystems towards oligotrophic communities more similar to the less diverse and less rich, and thus potentially less stable,
BATS summer microbial community (Giovannoni \& Vergin 2012). Thus, the finding that this community is less rich and depleted in rare taxa has implications for understanding the resilience of oceans to environmental perturbations in the future.

We also observed episodic population fluctuations in some taxa, particularly well-known copiotrophs, suggesting that the core time series data collected at BATS do not record some perturbations that influence the microbial community. Some NTUs, such as those belonging to Alteromonadaceae and Vibrionaceae, are well known from culturing and bottle experiments, but are rarely detected in lower-resolution molecular surveys (Suzuki et al. 1997, Eilers et al. 2000, McCarren et al. 2010). Previous work has demonstrated that additions of inorganic nitrogen or phosphorus alone or in combination are not sufficient to stimulate growth of populations of rare copiotrophs in the Sargasso Sea (Carlson et al. 2002). However, elevated macronutrient concentrations in combination with a labile dissolved organic carbon supply stimulate the production of these rare copiotrophs in vivo (Carlson et al. 2002, Mills et al. 2008, Nelson \& Carlson 2012). It is tempting to speculate that, at BATS, a labile molecule triggers episodic growth in these cases, but there is no direct evidence for this hypothesis. A comparison of the Vibrionaceae NTU distribution to pigment measurements suggests a link with chlorophyllide $a$, as both were anomalously high during winter 1998 (Fig. S3). However, it is unclear whether there was a direct connection between Vibrionaceae and chlorophyllide a. It is possible that labile compounds were directly released or were available as a result of top-down processes such as viral lysis or zooplankton feeding on abundant taxa. Despite the very large amount of data collected in the BATS time series, correlations that would explain the episodic abundance of the major copiotrophic taxa were elusive. This observation indicates that, at present, oceanographers may be missing events that are largely biological in nature that result in the release of labile dissolved organic material in the water column. These events might not necessarily be detected by total dissolved organic carbon measurements because of rapid turnover.

The historical context of the observations that we report about copiotrophs is important. These organisms (Vibrionaceae and Alteromonadaceae) were for many decades the most well studied marine bacteria because they could be cultured easily using common bacteriological methods such as agar plates (Baumann et al. 1972). We show that their abundance is typically low (average relative abundances of FA 
taxa ranged from 0.035 to $0.095 \%$ ), which is consistent with a perspective that has broadly emerged to explain the 'great plate count anomaly' (Staley \& Konopka 1985): plate counts accurately record the numbers of some very rare and unusual taxa that have a remarkable ability to exploit departures from ambient nutrient concentrations. The observations that we report here suggest that many rare taxa, like dominant taxa that have been the focus of many previous studies, are tightly entrained to environmental conditions and are unable to grow on agar plates. This suggests that, like dominant taxa, most rare taxa have a reduced metabolic repertoire that necessitates connections to other organisms through evolved nutrient dependencies (Tripp et al. 2008, Schwalbach et al. 2010, Sun et al. 2011, Carini et al. 2013).

In support of the conclusion that rapid growth to exploit episodic opportunities is a strategy used by a particular group of rare taxa (known as copiotrophs or R-strategists), but not others, we note that taxa that responded to episodic disturbances at BATS have among the shortest division times reported for microbial cells. For example, isolates belonging to Vibrionaceae doubled in as little as 12 min (Ulitzur 1974) while Alteromonas macleodii isolates doubled in 2.4 h (López-Pérez et al. 2012). Some have argued that organisms with short division times are more important in biogeochemical cycles, and that organisms with long division times succeed by being 'defense specialists' (Suttle 2007). However, low growth rates are normal in marine systems, and the product of mean growth rate and biomass determines the net impact of an organism. The best data available support the conclusion that organisms such as SAR11, that have slow growth rates (Rappé et al. 2002) and large population sizes (Morris et al. 2002), are much larger contributors to ocean biogeochemistry than copiotrophs (Malmstrom et al. 2004, Straza et al. 2010, Sun et al. 2011, Laghdass et al. 2012). Further support for this perspective came from a recent paper which reported that the phages of slowly growing SAR11 populations are the most abundant viruses in the oceans (Zhao et al. 2013), suggesting that SAR11 reproductive success is due to superior competition for ambient resources and not defense specialization.

The data presented here suggest that some bacterial taxa that are persistently rare or are detected episodically are responding to disturbances of unknown types. After winter mixing, richness was significantly higher and, in addition, as the mixed layer begins to deepen at the end of summer, rare taxa became more numerous, indicating that many persistently rare taxa are successful during periods characterized by trans- port by mixing and greater nutrient inputs. Some taxa, such as Vibrionaceae and Alteromonadaceae, occasionally reached maximal abundances many orders of magnitude higher than their median abundances, presumably in response to unknown disturbances. Thus, accurately modeling the responses of ocean ecosystems to disturbances may depend on knowing the growth rate and population density of rare taxa, and on developing new environmental measurements that record unrecognized environmental factors that cause these populations to fluctuate.

\section{SUMMARY}

With the improved sensitivity and precision of pyrosequencing methods, we have shown that spatiotemporal patterns such as seasonality and stratification, which are common in abundant bacterioplankton populations, also apply to most rare populations. These data support the perspective that, in most cases, rare members of the community have evolved and adapted to exploit fine variation in conditions and resources, leading to complex but reproducible patterns of microbial community turnover. These findings suggest that connectivity networks may be extended to include large numbers of rare taxa. In contrast, infrequent taxa, including most common culturable species (copiotrophs), attain population maxima in random patterns and appear to be much less connected. With further exploration of co-occurrence patterns in this dataset, we aim to explore these issues and shed light on the ecology of rare taxa in the dynamic western Sargasso Sea ecosystem.

Acknowledgements. We thank the officers and crew of the RV 'Weatherbird II' for their assistance and support and the BATS chief scientists and technicians for assistance in water collection and accommodating wire time requests. We thank R. Parsons, R. Poage, B. Beszteri, Z. Landry, M. Rappé, and D. Smith for technical assistance and J. Gilbert, C. Thrash, B. Temperton, K. Halsey, A. Thurber, and 3 reviewers for helpful suggestions. Major support was provided by grants from the Marine Microbiology Initiative of the Gordon and Betty Moore Foundation and the NSF Microbial Observatory program (MCB-0237713 \& OCE-0802004).

\section{LITERATURE CITED}

Baumann L, Baumann P, Mandel M, Allen RD (1972) Taxonomy of aerobic marine eubacteria. J Bacteriol 110: 402-429

Behrenfeld MJ (2010) Abandoning Sverdrup's critical depth hypothesis on phytoplankton blooms. Ecology 91: 977-989 
Behrenfeld MJ, O'Malley RT, Siegel DA, McClain CR and others (2006) Climate-driven trends in contemporary ocean productivity. Nature 444:752-755

Brinkmeyer R, Knittel K, Jürgens J, Weyland H, Amann R, Helmke E (2003) Diversity and structure of bacterial communities in Arctic versus Antarctic pack ice. Appl Environ Microbiol 69:6610-6619

- Brown MV, Schwalbach MS, Hewson I, Fuhrman JA (2005) Coupling 16S-ITS rDNA clone libraries and automated ribosomal intergenic spacer analysis to show marine microbial diversity: development and application to a time series. Environ Microbiol 7:1466-1479

> Campbell BJ, Yu L, Heidelberg JF, Kirchman DL (2011) Activity of abundant and rare bacteria in a coastal ocean. Proc Natl Acad Sci USA 108:12776-12781

Caporaso JG, Paszkiewicz K, Field D, Knight R, Gilbert JA (2012) The western English Channel contains a persistent microbial seed bank. ISME J 6:1089-1093

Carini P, Steindler L, Beszteri S, Giovannoni SJ (2013) Nutrient requirements for growth of the extreme oligotroph 'Candidatus Pelagibacter ubique' HTCC1062 on a defined medium. ISME J 7:592-602

- Carlson CA, Giovannoni SJ, Hansell DA, Goldberg SJ and others (2002) Effect of nutrient amendments on bacterioplankton production, community structure, and DOC utilization in the northwestern Sargasso Sea. Aquat Microb Ecol 30: 19-36

> Carlson CA, Giovannoni SJ, Hansell DA, Goldberg SJ, Parsons R, Vergin K (2004) Interactions among dissolved organic carbon, microbial processes, and community structure in the mesopelagic zone of the northwestern Sargasso Sea. Limnol Oceanogr 49:1073-1083

- Carlson CA, Morris R, Parsons R, Treusch AH, Giovannoni SJ, Vergin K (2009) Seasonal dynamics of SAR11 populations in the euphotic and mesopelagic zones of the northwestern Sargasso Sea. ISME J 3:283-295

Chao A (1984) Nonparametric-estimation of the number of classes in a population. Scand J Stat 11:265-270

Chao A, Shen TJ (2010) Program SPADE (Species Prediction And Diversity Estimation). Program and User's Guide. Available at http://chao.stat.nthu.edu.tw

Chapin FS III, Zavaleta ES, Eviner VT, Naylor RL and others (2000) Consequences of changing biodiversity. Nature 405:234-242

Clarke KR, Gorley RN (2006) PRIMER v6: user manual/ tutorial. PRIMER-E, Plymouth

> Daims H, Bruhl A, Amann R, Schleifer KH, Wagner M (1999) The domain-specific probe EUB338 is insufficient for the detection of all Bacteria: development and evaluation of a more comprehensive probe set. Syst Appl Microbiol 22: 434-444

> DuRand MD, Olson RJ, Chisholm SW (2001) Phytoplankton population dynamics at the Bermuda Atlantic Timeseries station in the Sargasso Sea. Deep-Sea Res II 48: 1983-2003

- Eilers H, Pernthaler J, Glockner FO, Amann R (2000) Culturability and in situ abundance of pelagic bacteria from the North Sea. Appl Environ Microbiol 66:3044-3051

Eppley RW, Peterson BJ (1979) Particulate organic matter flux and planktonic new production in the deep ocean. Nature 282:677-680

Fuhrman JA (2009) Microbial community structure and its functional implications. Nature 459:193-199

Fuhrman JA, Hewson I, Schwalbach MS, Steele JA, Brown MV, Naeem S (2006) Annually reoccurring bacterial communities are predictable from ocean conditions. Proc Natl Acad Sci USA 103:13104-13109

Galand PE, Casamayor EO, Kirchman DL, Lovejoy C (2009) Ecology of the rare microbial biosphere of the Arctic Ocean. Proc Natl Acad Sci USA 106:22427-22432

Gilbert JA, Steele JA, Caporaso JG, Steinbrück L and others (2012) Defining seasonal marine microbial community dynamics. ISME J 6:298-308

Giovannoni SJ, Rappé MS (2000) Evolution, diversity and molecular ecology of marine prokaryotes. In: Kirchman DL (ed) Microbial ecology of the oceans. Wiley-Liss, New York, NY, p 47-85

Giovannoni SJ, Vergin KL (2012) Seasonality in ocean microbial communities. Science 335:671-676

> Glasby TM, Underwood AJ (1996) Sampling to differentiate between pulse and press perturbations. Environ Monit Assess 42:241-252

- Gordon DA, Giovannoni SJ (1996) Stratified microbial populations related to Chlorobium and Fibrobacter detected in the Atlantic and Pacific Oceans. Appl Environ Microbiol 62:1171-1177

> Horner-Devine MC, Bohannan BJ (2006) Phylogenetic clustering and overdispersion in bacterial communities. Ecology 87(Suppl):S100-S108

$>$ Hugoni M, Taib N, Debroas D, Domaizon I and others (2013) Structure of the rare archaeal biosphere and seasonal dynamics of active ecotypes in surface coastal waters. Proc Natl Acad Sci USA 110:6004-6009

Jost L (2006) Entropy and diversity. Oikos 113:363-375

Jost L (2010) The relation between evenness and diversity. Diversity 2:207-232

$>$ Keddy PA (1992) Assembly and response rules - two goals for predictive community ecology. J Veg Sci 3:157-164

Koeppel AF, Wu M (2013) Surprisingly extensive mixed phylogenetic and ecological signals among bacterial Operational Taxonomic Units. Nucleic Acids Res 41: $5175-5188$

> Laghdass M, Catala P, Caparros J, Oriol L, Lebaron P, Obernosterer I (2012) High contribution of SAR11 to microbial activity in the North West Mediterranean Sea. Microb Ecol 63:324-333

Lennon JT, Jones SE (2011) Microbial seed banks: the ecological and evolutionary implications of dormancy. Nat Rev Microbiol 9:119-130

Letunic I, Bork P (2011) Interactive Tree Of Life v2: online annotation and display of phylogenetic trees made easy. Nucleic Acids Res 39:W475-478

López-Pérez M, Gonzaga A, Martin-Cuadrado AB, Onyshchenko O, Ghavidel A, Ghai R, Rodriguez-Valera F (2012) Genomes of surface isolates of Alteromonas macleodii: the life of a widespread marine opportunistic copiotroph. Sci Rep 2:696

> Malmstrom RR, Kiene RP, Cottrell MT, Kirchman DL (2004) Contribution of SAR11 bacteria to dissolved dimethylsulfoniopropionate and amino acid uptake in the North Atlantic ocean. Appl Environ Microbiol 70:4129-4135

Matsen FA, Kodner RB, Armbrust EV (2010) pplacer: linear time maximum-likelihood and Bayesian phylogenetic placement of sequences onto a fixed reference tree. BMC Bioinformatics 11:538

> McCarren J, Becker JW, Repeta DJ, Shi Y and others (2010) Microbial community transcriptomes reveal microbes and metabolic pathways associated with dissolved organic matter turnover in the sea. Proc Natl Acad Sci USA 107:16420-16427 
Menzel DW, Ryther JH (1960) The annual cycle of primary production in the Sargasso Sea off Bermuda. Deep-Sea Res 6:351-367

Mills MM, Moore CM, Langlois R, Milne A and others (2008) Nitrogen and phosphorus co-limitation of bacterial productivity and growth in the oligotrophic subtropical North Atlantic. Limnol Oceanogr 53:824-834

> Morin PJ, McGrady-Steed J (2004) Biodiversity and ecosystem functioning in aquatic microbial systems: a new analysis of temporal variation and species richness-predictability relations. Oikos 104:458-466

Morris RM, Rappé MS, Connon SA, Vergin KL, Siebold WA, Carlson CA, Giovannoni SJ (2002) SAR11 clade dominates ocean surface bacterioplankton communities. Nature 420:806-810

Morris RM, Rappé MS, Urbach E, Connon SA, Giovannoni SJ (2004) Prevalence of the Chloroflexi-related SAR202 bacterioplankton cluster throughout the mesopelagic zone and deep ocean. Appl Environ Microbiol 70: 2836-2842

Morris RM, Vergin KL, Cho JC, Rappé MS, Carlson CA, Giovannoni SJ (2005) Temporal and spatial response of bacterioplankton lineages to annual convective overturn at the Bermuda Atlantic Time-series Study site. Limnol Oceanogr 50:1687-1696

Morris JJ, Lenski RE, Zinser ER (2012) The Black Queen Hypothesis: evolution of dependencies through adaptive gene loss. mBio 3:e00036-12

Müller AH, Gough SP, Bollivar DW, Meldal M, Willows RD, Hansson M (2011) Methods for the preparation of chlorophyllide $a$ : an intermediate of the chlorophyll biosynthetic pathway. Anal Biochem 419:271-276

> Naeem S, Li SB (1997) Biodiversity enhances ecosystem reliability. Nature 390:507-509

> Neef A, Amann R, Schlesner H, Schleifer KH (1998) Monitoring a widespread bacterial group: in situ detection of planctomycetes with $16 \mathrm{~S}$ rRNA-targeted probes. Microbiology 144:3257-3266

Nelson CE, Carlson CA (2012) Tracking differential incorporation of dissolved organic carbon types among diverse lineages of Sargasso Sea bacterioplankton. Environ Microbiol 14:1500-1516

> Nicholson DP, Stanley RHR, Barkan E, Karl DM, Luz B, Quay PD, Doney SC (2012) Evaluating triple oxygen isotope estimates of gross primary production at the Hawaii Ocean Time-series and Bermuda Atlantic Time-series Study sites. J Geophys Res 117, C05012 doi:10.1029/ 2010JC006856

Pedrós-Alió C (2006) Marine microbial diversity: Can it be determined? Trends Microbiol 14:257-263

> Pedrós-Alió C (2012) The rare bacterial biosphere. Annu Rev Mar Sci 4:449-466

> Prosser JI, Bohannan BJ, Curtis TP, Ellis RJ and others (2007) The role of ecological theory in microbial ecology. Nat Rev Microbiol 5:384-392

Rappé MS, Connon SA, Vergin KL, Giovannoni SJ (2002) Cultivation of the ubiquitous SAR11 marine bacterioplankton clade. Nature 418:630-633

Editorial responsibility: Jed Fuhrman,

Los Angeles, California, USA
Schwalbach MS, Tripp HJ, Steindler L, Smith DP, Giovannoni SJ (2010) The presence of the glycolysis operon in SAR11 genomes is positively correlated with ocean productivity. Environ Microbiol 12:490-500

Sogin ML, Morrison HG, Huber JA, Mark Welch D and others (2006) Microbial diversity in the deep sea and the underexplored 'rare biosphere'. Proc Natl Acad Sci USA 103:12115-12120

Sprintall J, Tomczak M (1992) Evidence of the barrier layer in the surface-layer of the tropics. J Geophys Res 97: 7305-7316

Staley JT, Konopka A (1985) Measurement of in situ activities of nonphotosynthetic microorganisms in aquatic and terrestrial habitats. Annu Rev Microbiol 39:321-346

> Steinberg DK, Carlson CA, Bates NR, Johnson RJ, Michaels AF, Knap AH (2001) Overview of the US JGOFS Bermuda Atlantic Time-series Study (BATS): a decadescale look at ocean biology and biogeochemistry. DeepSea Res II 48:1405-1447

- Straza TRA, Ducklow HW, Murray AE, Kirchman DL (2010) Abundance and single-cell activity of bacterial groups in Antarctic coastal waters. Limnol Oceanogr 55:2526-2536

Sun J, Steindler L, Thrash JC, Halsey KH and others (2011) One carbon metabolism in SAR11 pelagic marine bacteria. PLoS ONE 6:e23973

Suttle CA (2007) Marine viruses - major players in the global ecosystem. Nat Rev Microbiol 5:801-812

Suzuki MT, Rappé MS, Haimberger ZW, Winfield H, Adair N, Ströbel J, Giovannoni SJ (1997) Bacterial diversity among small-subunit rRNA gene clones and cellular isolates from the same seawater sample. Appl Environ Microbiol 63:983-989

Treusch AH, Vergin KL, Finlay LA, Donatz MG, Burton RM, Carlson CA, Giovannoni SJ (2009) Seasonality and vertical structure of microbial communities in an ocean gyre. ISME J 3:1148-1163

> Treusch AH, Demir-Hilton E, Vergin KL, Worden AZ and others (2012) Phytoplankton distribution patterns in the northwestern Sargasso Sea revealed by small subunit rRNA genes from plastids. ISME J 6:481-492

> Tripp HJ, Kitner JB, Schwalbach MS, Dacey JW, Wilhelm LJ, Giovannoni SJ (2008) SAR11 marine bacteria require exogenous reduced sulphur for growth. Nature 452 : $741-744$

Ulitzur S (1974) Vibrio parahaemolyticus and Vibrio alginolyticus: short generation-time marine bacteria. Microb Ecol 1:127-135

Vergin KL, Beszteri B, Monier A, Thrash JC and others (2013) High-resolution SAR11 ecotype dynamics at the Bermuda Atlantic Time-series Study site by phylogenetic placement of pyrosequences. ISME J 7:1322-1332

Wittebolle L, Marzorati M, Clement L, Balloi A and others (2009) Initial community evenness favours functionality under selective stress. Nature 458:623-626

- Zhao Y, Temperton B, Thrash JC, Schwalbach MS and others (2013) Abundant SAR11 viruses in the ocean. Nature 494:357-360

Submitted: May 22, 2013; Accepted: September 5, 2013 Proofs received from author(s): November 6, 2013 